\title{
Immunomodulatory actions of a kynurenine-derived endogenous electrophile.
}

Mara Carreño $^{1, \dagger}$, Maria F. Pires ${ }^{1, \dagger}$, Steven R. Woodcock ${ }^{1}$, Tomasz Brzoska ${ }^{2,3}$, Samit Ghosh ${ }^{2,3}$, Sonia R. Salvatore ${ }^{1}$, Matthew Dunn ${ }^{1}$, Nora Connors ${ }^{1}$, Shuai Yuan ${ }^{2}$, Adam C. Straub ${ }^{1,2,4}$, Stacy G. Wendell $^{1,8}$, Gregory J. Kato ${ }^{5}$, Bruce A. Freeman ${ }^{1}$, Solomon Ofori-Acquah ${ }^{2,3,6}$, Prithu Sundd ${ }^{2,3,7}$, Francisco J. Schopfer ${ }^{1,2,8}$, Dario A. Vitturi ${ }^{1,2,9^{*}}$.

${ }^{1}$ Department of Pharmacology and Chemical Biology, University of Pittsburgh; Pittsburgh, Pennsylvania, USA.

${ }^{2}$ Pittsburgh Heart, Lung and Blood Vascular Medicine Institute, University of Pittsburgh; Pittsburgh, Pennsylvania, USA.

${ }^{3}$ Division of Hematology/Oncology, Department of Medicine, University of Pittsburgh; Pittsburgh, Pennsylvania, USA.

${ }^{4}$ Center for Microvascular Research, University of Pittsburgh; Pittsburgh, Pennsylvania, USA.

${ }^{5}$ CSL Behring; King of Prussia, Pennsylvania, USA.

${ }^{6}$ School of Biomedical and Allied Health Sciences, University of Ghana; Accra, Ghana.

${ }^{7}$ Department of Bioengineering, University of Pittsburgh; Pittsburgh, Pennsylvania, USA.

${ }^{8}$ Pittsburgh Liver Research Center, University of Pittsburgh; Pittsburgh, Pennsylvania, USA.

${ }^{9}$ Center for Critical Care Nephrology, University of Pittsburgh; Pittsburgh, Pennsylvania, USA.

*Corresponding author. Email: dav28@ pitt.edu

${ }^{\dagger}$ These authors contributed equally to this work.

\begin{abstract}
The inflammatory upregulation of kynurenine metabolism induces immunomodulatory responses via incompletely understood mechanisms. We report that increases in cellular and systemic kynurenine levels yield the electrophilic derivative kynurenine-carboxyketoalkene (Kyn-CKA), as evidenced by the accumulation of thiol-conjugates and saturated metabolites. Under physiological conditions, Kyn-CKA induces Nrf2-regulated genes and inhibits NF- $\mathrm{BB}$ and NLRP3-dependent pro-inflammatory signaling. Sickle Cell Disease (SCD) is a hereditary hemolytic condition characterized by basal inflammation and recurrent vaso-occlusive crises. Both a transgenic SCD murine model and SCD patients exhibit increased kynurenine synthesis and elevated Kyn-CKA metabolite levels. Plasma hemin and kynurenine concentrations are positively correlated, indicating that Kyn-CKA synthesis in SCD is upregulated during pathogenic vascular stress. Remarkably, exogenous administration of Kyn-CKA abrogated pulmonary microvasculature occlusion in SCD mice, an important factor in the development of lung injury. These findings demonstrate that the upregulation of kynurenine synthesis and its metabolism to Kyn-CKA is an adaptive response that attenuates inflammation and protects tissues.
\end{abstract}

One-Sentence Summary: Kyn-CKA is a kynurenine-derived signaling mediator that transduces its immunomodulatory protective actions and attenuates vaso-occlusion in sickle cell disease. 


\section{Introduction}

Acute and chronic inflammation induces the rate-limiting enzymes indoleamine dioxygenase-1 (IDO1) and tryptophan dioxygenase-2 (TDO2) that in turn increase kynurenine and kynurenine/tryptophan ratios (1). Increased kynurenine synthesis via TDO2 in hepatocytes is protective against primary endotoxemia, while IDO1 induction in myeloid leukocytes attenuates inflammatory responses to endotoxin, promotes the generation of tolerogenic dendritic cells (DC) and facilitates $\mathrm{T}_{\text {reg }}$ polarization (2-5). Consistent with these immunomodulatory effects, dysregulated kynurenine metabolism occurs in inflammatory conditions such as cardiovascular disease, cancer, renal injury, transplantation and is often a strong predictor of outcome (6-9). However, despite its pathophysiological relevance, the mechanisms responsible for the immunoregulatory effects of kynurenine and its metabolites are poorly defined (10).

Biological electrophiles contain carbon-carbon double bonds conjugated to electronwithdrawing moieties that generate electron-deficient carbons that are susceptible to nucleophilic attack by thiols or, less predominantly, amines (11). Covalent modification of critical cysteine thiols in transcription factors, signaling proteins, and metabolic enzymes have profound effects on cellular function (11). In particular, reactive electrophiles elicit cytoprotective and immunomodulatory effects both preclinically and clinically by upregulating Nrf2-dependent antioxidant enzyme translation and inhibiting NF- $\mathrm{BB}-$ dependent gene expression and inflammatory cell metabolic reprogramming $(11,12)$. In vivo, kynurenine deamination generates an $\alpha, \beta$-unsaturated carbonyl-containing product, Kynurenine-Carboxy-Keto-Alkene (KynCKA), which participates in the generation of UV-filters in the eye lens (13). Critically, the potential for Kyn-CKA to contribute to the immunomodulatory actions of kynurenine metabolites has not been explored.

Herein, we show that kynurenine synthesis in hepatocytes and macrophages generates Kyn-CKA. Novel Kyn-CKA-specific metabolites detected in healthy humans and mice affirms that Kyn-CKA formation is biologically significant, with Kyn-CKA generation upregulated by increases in systemic kynurenine concentrations. Kyn-CKA activates Nrf2-dependent gene expression and inhibits NF- $\mathrm{KB}$ and NLRP3-dependent pro-inflammatory responses in both parenchymal and immune cells. These responses are recapitulated across several tissues in vivo. In addition to upregulating Nrf2-dependent signaling under basal non-activated conditions, Kyn-CKA attenuates LPS-induced pro-inflammatory gene expression and cytokine synthesis. Finally, Kyn-CKA increases IL-10 levels and attenuates the expression of renal injury markers in LPS-treated mice, suggesting potent anti-inflammatory and cytoprotective actions in vivo.

The anti-inflammatory effects of Kyn-CKA suggested the possibility that its formation might constitute a missing link in kynurenine signaling and an adaptive immunomodulatory response having the potential to modulate disease progression. To test this hypothesis, we focused on the hemolytic inflammatory conditions intrinsic to Sickle Cell Disease (SCD), wherein the activation of endothelial, platelet and leukocyte Toll-like Receptor-4 (TLR4) by hemoglobin-derived products promotes the formation of multicellular aggregates which, in combination with increased sickle erythrocyte rigidity and adhesiveness, cause vaso-occlusion and ischemic injury. This phenomenon, not only causes severe pain crises but also, leads to the development of lifethreatening acute and chronic complications in SCD patients $(14,15)$. Analysis of plasma samples obtained from a transgenic humanized SCD mouse model and from SCD patients showed elevated kynurenine levels which correlated positively with pro-inflammatory plasma hemin concentrations. This increased kynurenine synthesis was further correlated with greater Kyn-CKA metabolite concentrations in the plasma and urine of SCD mice and SCD patients. 
Quantitative fluorescence intravital microscopy was used to monitor vaso-occlusive occurrence in the lung microvasculature of SCD mice and to test the role of Kyn-CKA as a modulator of tissue dysfunction $(16,17)$. Consistent with a role Kyn-CKA as an endogenous antiinflammatory mediator in SCD, Kyn-CKA significantly decreased the average size of multicellular aggregates and inhibited vaso-occlusion of the pulmonary microvasculature.

Collectively, our results reveal that Kyn-CKA is present under basal conditions and that its formation is adaptively elevated within the context of inflammatory kynurenine synthesis upregulation. Kyn-CKA is an endogenous electrophilic signaling mediator capable of attenuating pro-inflammatory signaling cascades, modulating complex pathological responses, and potentially, impacting the course of human disease.

\section{Results}

Kynurenine yields Kyn-CKA and promotes Nrf2-dependent gene expression. Kynurenine is a main product of tryptophan (Trp) metabolism and, upon deamination, is activated into Kyn-CKA, an $\alpha, \beta$-unsaturated carbonyl-containing derivative with electrophilic reactivity (Fig 1A). Treatment of the normal mouse hepatocyte cell line AML-12 with Kyn-CKA dosedependently upregulated electrophile-sensitive Nrf2-dependent gene expression both at the transcript and protein levels, suggesting that Kyn-CKA is a chemically and biologically active product (Fig 1B,C). Consistent with its electrophilic reactivity, Kyn-CKA rapidly targeted intracellular thiols, as evidenced by the formation of glutathione (GSH) conjugates (GSH-KynCKA) that were excreted and detected in the extracellular milieu (Fig 1D). To define the contribution of intracellular enzymatic reactions to the endogenous formation of Kyn-CKA, AML-12 were treated with Trp and the levels of intermediate metabolites N-formyl-kynurenine and kynurenine were assessed. Trp caused a significant increase in $\mathrm{N}$-formyl-kynurenine and kynurenine synthesis that led to the formation of GSH-Kyn-CKA adducts (Fig 1E-G). Similarly, treatment with N-formyl-kynurenine (NF-Kyn) or L-kynurenine (L-Kyn) resulted in dosedependent GSH-Kyn-CKA formation (Fig 1H). The formation of GSH conjugates is the first step in the mercapturic acid pathway, a conserved mechanism for cellular electrophile inactivation that is followed by export through multidrug resistance proteins (MRP). Consistent with this pathway being engaged in Kyn-CKA metabolism, treatment with the pan-MRP inhibitor probenecid induced a significant increase in intracellular GSH-Kyn-CKA levels (Fig 1I). Finally, and parallel to the endogenous formation of GSH-Kyn-CKA adducts, treatment of AML-12 cells with Trp significantly induced Keap1-regulated Nrf2-dependent gene expression (Fig 1J). This underscores that intracellular Kyn-CKA formation can evade both high cellular GSH concentrations and trapping by the mercapturic acid pathways to induce Keap1 thiol alkylation and Nrf2-regulated signaling. Consistent with its high electrophilic reactivity, non-thiol adducted Kyn-CKA was not detectable in both control and Trp-treated cells (not shown). 


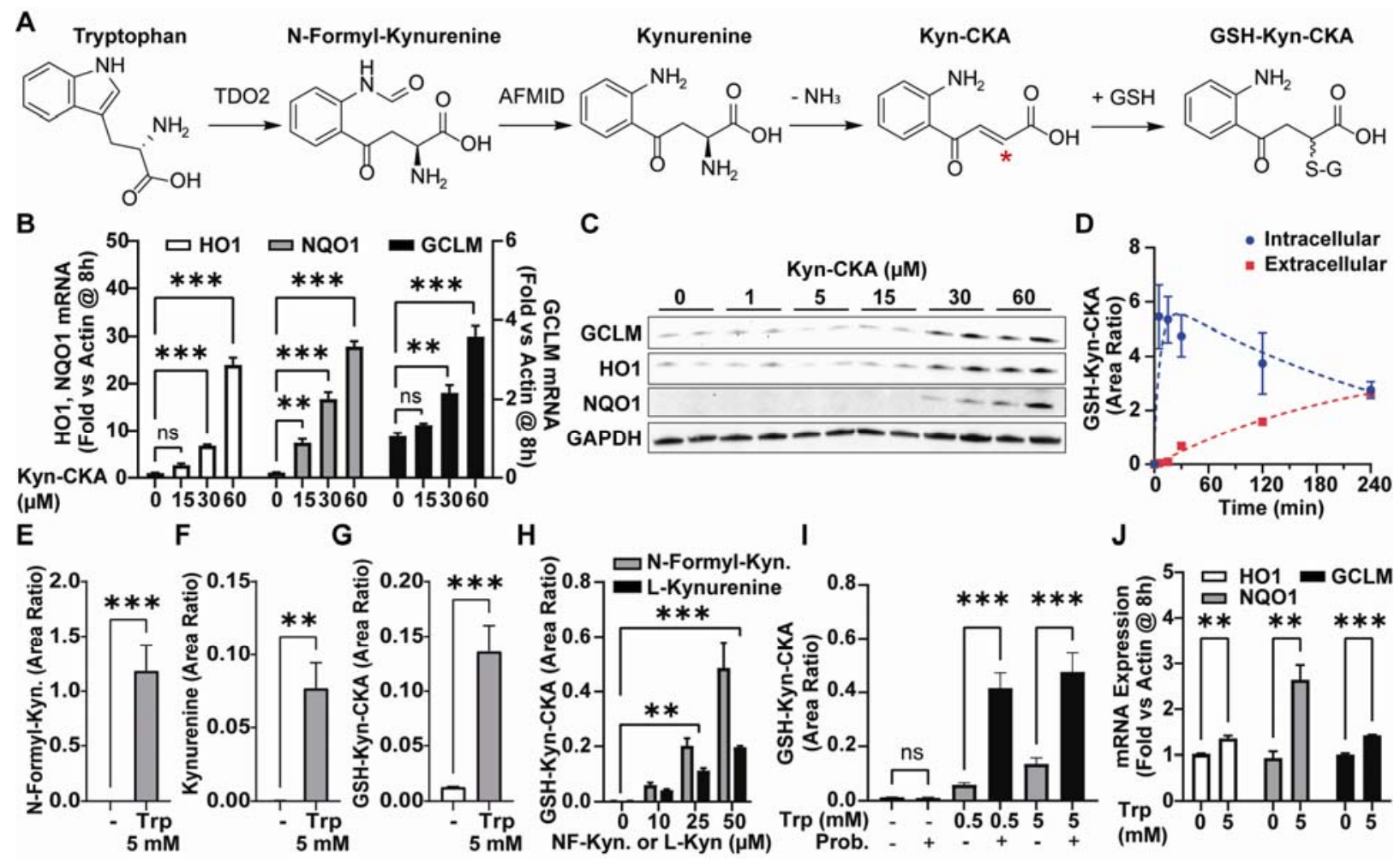

Fig. 1. Bioactive Kyn-CKA is formed following kynurenine synthesis in AML-12 cells. (A) Proposed route for Kyn-CKA formation following from Trp metabolism. The asterisk denotes the electrophilic carbon. (B) Nrf2-dependent gene induction by Kyn-CKA (n=3). (C) Nrf2regulated protein expression $24 \mathrm{~h}$ post Kyn-CKA. (D) GSH-Kyn-CKA formation following Kyn-CKA treatment $(60 \mu \mathrm{M}, \mathrm{n}=2$ per time point). Data is expressed as the ratio between GSHKyn-CKA and Trp- $\mathrm{d}_{3}$ areas. (E-G) Kynurenine metabolites $8 \mathrm{~h}$ post $\operatorname{Trp}(5 \mathrm{mM}, \mathrm{n}=3)$. (H) GSHKyn-CKA formation $8 \mathrm{~h}$ post NF-Kyn or L-Kyn ( $\mathrm{n}=2$ per dose). (I) Effect of probenecid $(1 \mathrm{mM})$ on GSH-Kyn-CKA after Trp for $8 \mathrm{~h}(\mathrm{n}=3)$. (J) Nrf2-dependent gene expression $8 \mathrm{~h}$ post Trp $(\mathrm{n}=3) . * * \mathrm{p}<0.01, * * * \mathrm{p}<0.0001$ by one- $(\mathrm{B})$ or two-way ANOVA and Tuckey's test (I), or by t-test $(\mathrm{E}-\mathrm{H}, \mathrm{J})$.

In vivo generation of Kyn-CKA. In vivo, intracellular electrophile-GSH conjugates are exported and metabolized to yield cysteine and mercapturic acid conjugates that are excreted in urine. To evaluate Kyn-CKA-specific metabolites in vivo, urine was collected from C57B1/6J mice either in the absence or $8 \mathrm{~h}$ following Kyn-CKA supplementation $(20,50 \mathrm{mg} / \mathrm{kg}$ ip). Consistent with its endogenous nature, LC-MS/MS analyses demonstrated the presence of urinary Cys-Kyn-CKA adducts in untreated mice which was confirmed by co-elution with a synthetic ${ }^{13} \mathrm{C}_{3}$ - Cys-KynCKA standard (Fig 2A). Exogenous Kyn-CKA administration gave higher levels of urinary Cys-Kyn-CKA adducts and led to the excretion of $\mathrm{N}$-acetyl-cysteine Kyn-CKA conjugates (NAC-Kyn-CKA, Fig 2A,B and Suppl Fig 1). In addition, liver tissue analysis revealed endogenous GSH-Kyn-CKA adducts in untreated mice, with GSH-Kyn-CKA significantly increased by Kyn-CKA treatment (Fig 2C,D and Suppl Fig 1). These results were recapitulated in $\mathrm{C} 57 \mathrm{~B} 1 / 6 \mathrm{~J}$ treated with L-Kyn, suggesting that systemic increases in this amino acid metabolite can promote Kyn-CKA formation in vivo (Fig 2E,F). Untargeted analysis of plasma samples from Kyn-CKA-treated mice revealed unadducted Kyn-CKA together with several upregulated LC-MS/MS features, including a reduced carboxyketoalkane derivative termed Red-Kyn-CKA 
(Fig 2G, Suppl Fig 2). Red-Kyn-CKA formation from Kyn-CKA was validated using a rat liver S9 fraction plus NADPH as a source of reducing equivalents (Suppl Fig 2) and confirmed by comparing the $\mathrm{MS}^{2}$ fragmentation pattern with a synthetic standard (Fig 2H). Unlike urinary and hepatic conjugates, neither free Kyn-CKA nor Red-Kyn-CKA were detected in untreated mouse plasma (not shown). Transcript expression analysis from Kyn-CKA treated mice revealed induction of Nrf2-dependent signaling in kidneys, heart, and liver (Fig 2I-K) and confirmed at the protein level in liver and kidneys (Fig 2L,M). No changes were observed in lung and brain (not shown). Nrf2-dependent gene expression was also upregulated in the liver of L-Kyn treated mice, consistent with Kyn-CKA formation in this tissue (Fig $2 \mathrm{~N}$ ) and reinforced by both increased levels of hepatic GSH-Kyn-CKA and urinary Cys-Kyn-CKA conjugates (Fig 2E,F).

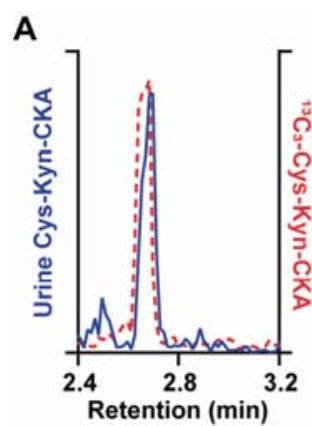

E

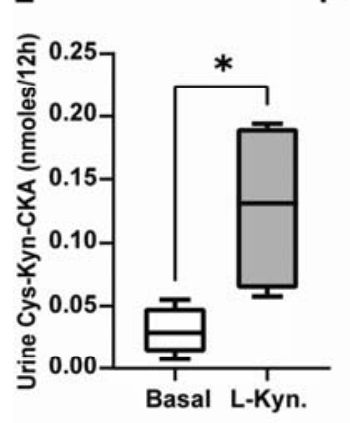

$\mathbf{F}$
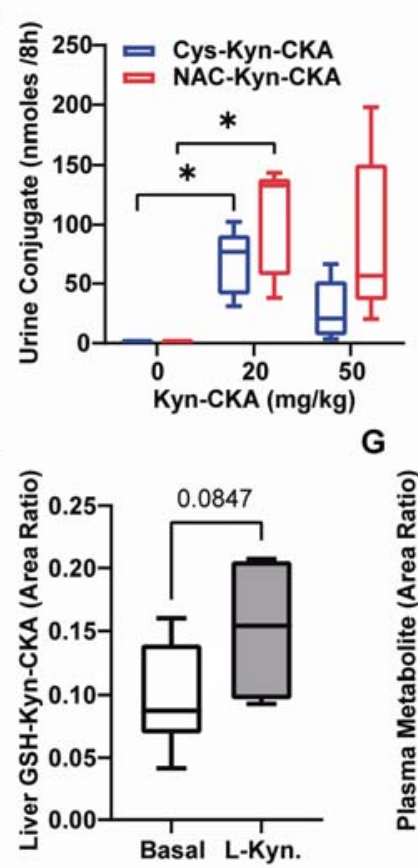

G
C

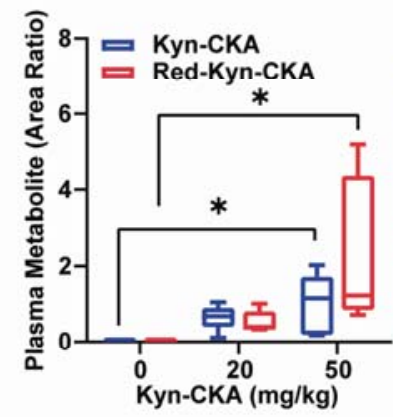

Heart

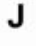

$\mathbf{J}$

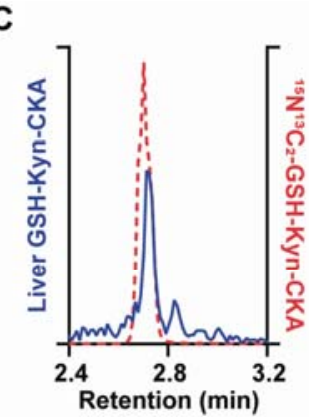

H
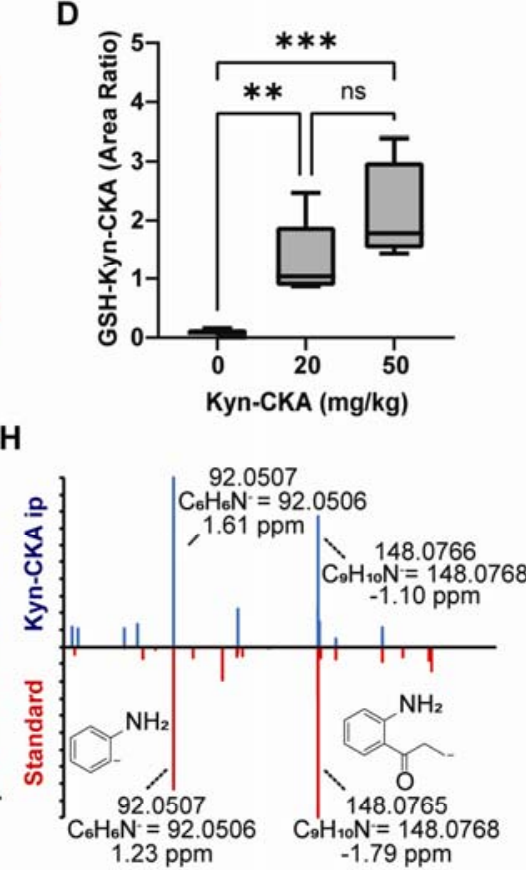

K

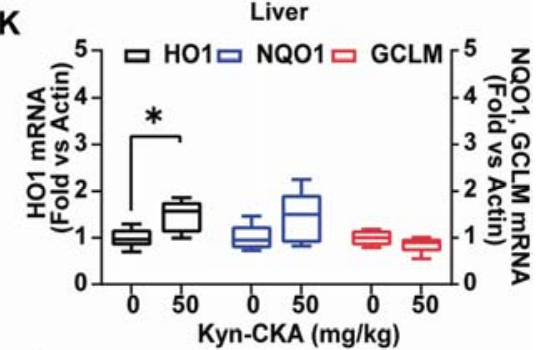

M

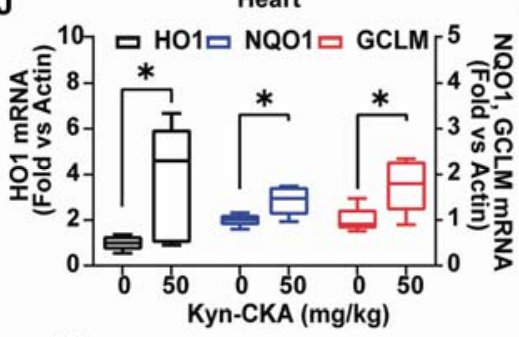

N

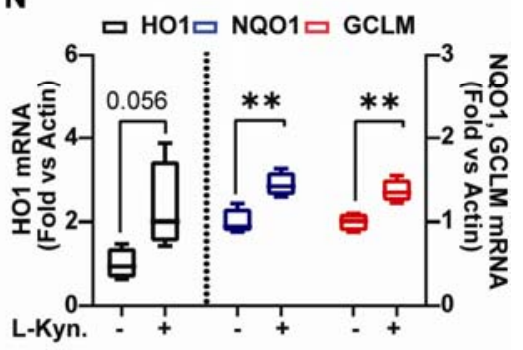

Fig. 2. Kyn-CKA is formed endogenously and induces Nrf2-dependent genes in vivo. (A) Representative LC-MS/MS trace showing co-elution between endogenous urinary Cys-Kyn- 
CKA and an isotopically labeled synthetic standard. (B) Urine Cys- and NAC-Kyn-CKA $8 \mathrm{~h}$ post Kyn-CKA (ip) in C57Bl6/J mice ( $\mathrm{n}=5$ per dose). (C) LC-MS/MS trace of endogenous GSHKyn-CKA in untreated mouse liver. (D) Hepatic GSH-Kyn-CKA in mice $8 \mathrm{~h}$ following Kyn-CKA (basal $n=9, n=5$ per dose). (E) Urinary Cys-Kyn-CKA in mice receiving L-Kyn (100 $\mathrm{mg} / \mathrm{kg}$ ip, $2 \mathrm{~d}, \mathrm{n}=4)$. (F) Hepatic GSH-Kyn-CKA L-Kyn treated mice (100 mg/kg ip, 2 d, basal $\mathrm{n}=9$, L-Kyn $\mathrm{n}=4$ ). (G) Plasma Kyn-CKA metabolites $8 \mathrm{~h}$ post-dosing. (H) Red-Kyn-CKA MS ${ }^{2}$ spectra for Kyn-CKA treated plasma (20 mg/kg, above) and synthetic Red-Kyn-CKA (below). (I-K) Nrf2-dependent gene expression in mice $8 \mathrm{~h}$ post Kyn-CKA $(50 \mathrm{mg} / \mathrm{kg}, \mathrm{n}=5$ per dose). (L, M) Nrf2-dependent protein levels in mice $24 \mathrm{~h}$ post Kyn-CKA (20 mg/kg ip, 2 doses, $8 \mathrm{~h}$ apart). (N) Nrf2-dependent gene expression in mice $12 \mathrm{~h}$ post second L-Kyn dose $(50 \mathrm{mg} / \mathrm{kg}$ ip, 2 doses, $24 \mathrm{~h}$ apart, $\mathrm{n}=4) . * \mathrm{p}<0.05, * * \mathrm{p}<0.01, * * * \mathrm{p}<0.0001$ by one-way ANOVA and Tukey's test $(\mathrm{B}, \mathrm{D}, \mathrm{G})$ or t-test $(\mathrm{E}, \mathrm{F}, \mathrm{I}-\mathrm{N})$. Kidney HO-1 in (M) was analyzed by Mann-Whitney test.

\section{Kyn-CKA inhibits NF- KB-dependent signaling and NLRP3 inflammasome engagement in} macrophages. While TDO2 catalyzes kynurenine synthesis in the liver, this reaction is mediated by IDO1 in myeloid cells. Akin to the results obtained with AML12 hepatocytes, Trp treatment of J774a.1 macrophages increased the formation of $\mathrm{N}$-formyl-kynurenine and kynurenine as well as GSH-Kyn-CKA (Fig 3A,C). In addition to inducing Nrf2-dependent gene expression (Suppl. Fig 3A), Kyn-CKA inhibited the LPS-induced expression of pro-inflammatory cytokines and enzymes that generate nitrogen oxides in both J774a.1 (Fig 3D-H) and bone marrow-derived macrophages (BMDM, Suppl. Fig 3B,C). NF- $\kappa$ B activation is essential in priming the NLRP3 inflammasome via increased synthesis of NLRP3 subunits and pro-IL-1 $\beta$. Activation of the primed inflammasome promotes proteolytic pro-caspase- 1 activation followed by mature IL-1 $\beta$ generation (12). Kyn-CKA treatment of LPS/ATP activated J774a.1 dose-dependently inhibited NLRP3 and pro-IL1 $\beta$ expression and attenuated pro-caspase-1 levels to inhibit processing and secretion of mature IL-1 $\beta$ (Fig 3I), with similar results obtained using BMDM (Suppl Fig 3D). Beyond an effect on myeloid cells, Kyn-CKA inhibited LPS-induced VCAM-1 expression in human pulmonary microvascular endothelial cells (HPMVEC), supporting that Kyn-CKA can invoke systemic anti-inflammatory responses across different cell types and tissues (Fig 3J).
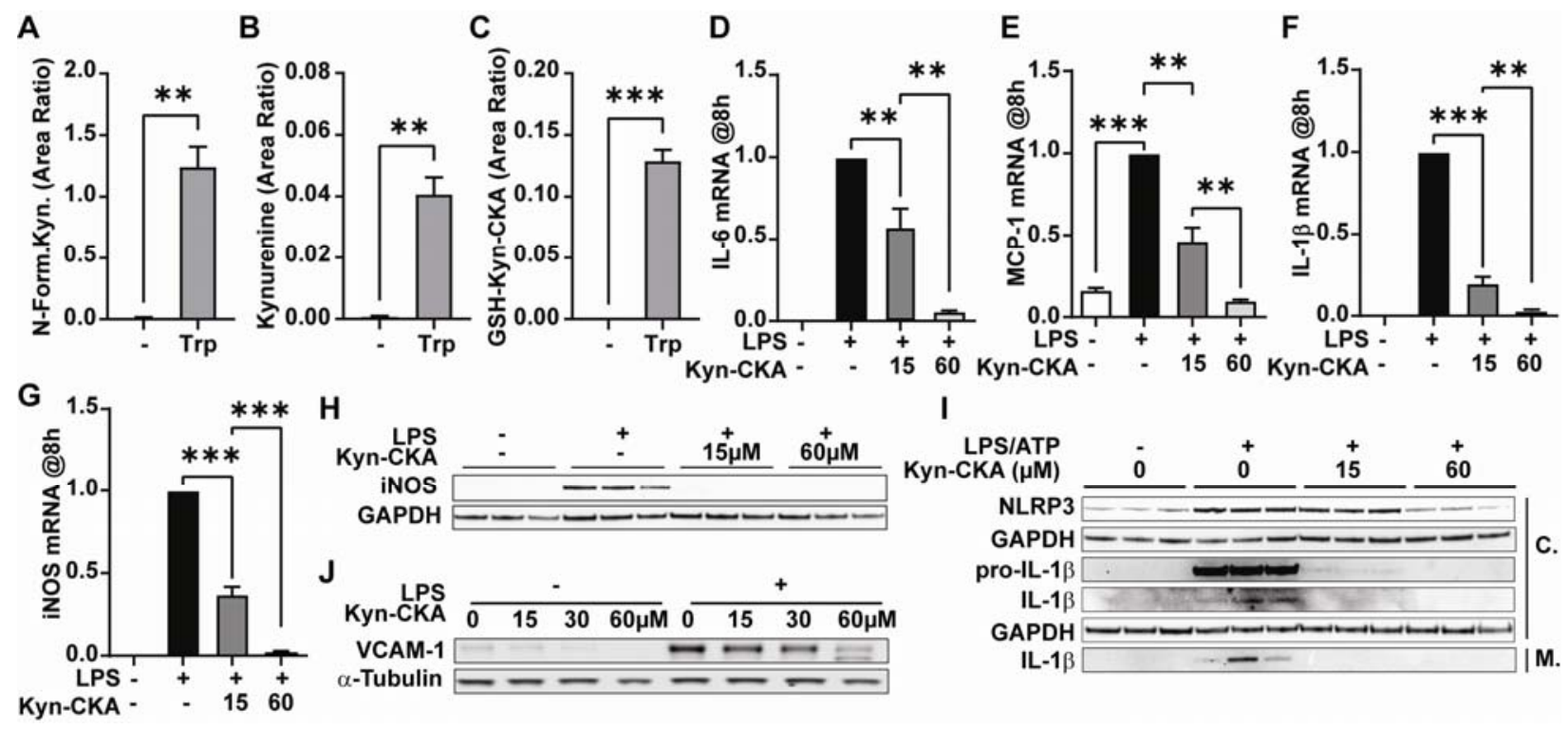

I

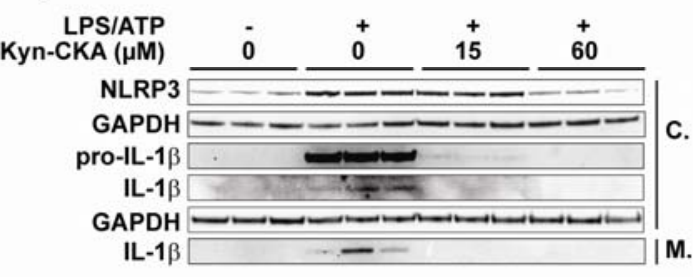


Fig. 3. Kyn-CKA is formed and attenuates NF-kB and NLRP3 signaling in macrophages. (A-C) Kynurenine metabolites in J774a.1 $4 \mathrm{~h}$ post Trp $(5 \mathrm{mM}, \mathrm{n}=3)$. (D-G) NF- $\kappa \mathrm{B}$-dependent gene expression $8 \mathrm{~h}$ post LPS $(100 \mathrm{ng} / \mathrm{mL})$ and Kyn-CKA $(15$ or $60 \mu \mathrm{M}, \mathrm{n}=3)$. (H) iNOS expression $8 \mathrm{~h}$ post LPS (100 $\mathrm{ng} / \mathrm{mL})$ and Kyn-CKA. (I) Inhibition of NLRP3 inflammasome activation by LPS/ATP (100 ng/mL, ATP $2 \mathrm{mM})$ by Kyn-CKA at $8 \mathrm{~h}$. C: Cellular fraction, M: Media. (J) VCAM-1 expression in HPMVEC $16 \mathrm{~h}$ post LPS $(100 \mathrm{ng} / \mathrm{mL})$ and Kyn-CKA. ** $\mathrm{p}<$ $0.01, * * * \mathrm{p}<0.0001$ by t-test (A-C) or one-way ANOVA and Tukey's test (D-G).

To determine whether the anti-inflammatory effects of Kyn-CKA in vitro translate to in vivo inflammatory scenarios, C57B16/J mice were treated with a single dose of Kyn-CKA (50 mg/kg

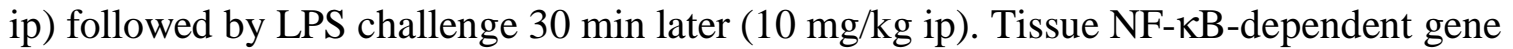
expression revealed anti-inflammatory responses to Kyn-CKA in kidneys (Fig 4A-D), heart (Fig 4E-H), and lung (Fig 4I). Kyn-CKA pretreatment also decreased the expression of the renal injury marker Kim-1, affirming attenuation of LPS-induced acute kidney injury (Fig 4J). Consistent with the induction of systemic anti-inflammatory responses, Kyn-CKA significantly inhibited plasma levels of several pro-inflammatory cytokines (Fig 4K-O) while increasing the anti-inflammatory mediator IL-10 (Fig 4P). 

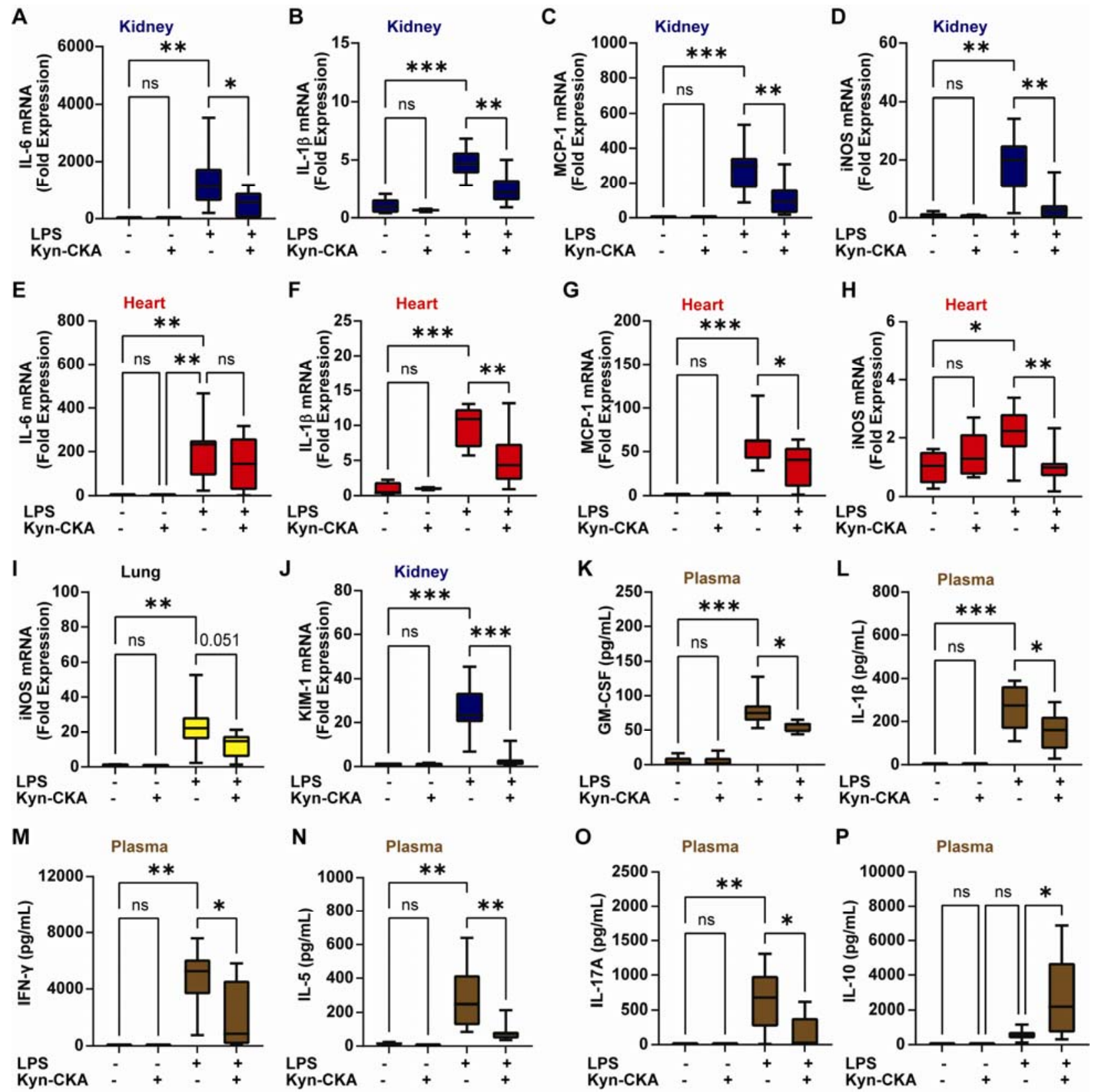

Fig. 4. Kyn-CKA promotes anti-inflammatory actions in vivo. (A-I) Kyn-CKA (50 mg/kg ip, $30 \mathrm{~min}$ before challenge) inhibits NF- KB target expression $8 \mathrm{~h}$ post LPS (10 $\mathrm{mg} / \mathrm{kg}$ ip). (J) KynCKA inhibits $8 \mathrm{~h}$ Kim-1 following LPS. (K-P) Plasma cytokine levels modulated by Kyn-CKA 8 h post-LPS. Non-LPS treated ( $n=5)$, LPS-treated $(n=8-9)$. $* \mathrm{p}<0.05, * * p<0.01, * * * p<0.001$ by one-way ANOVA and Tukey's test.

\section{Kyn-CKA levels are elevated in SCD and Kyn-CKA administration attenuates inflammatory} vaso-occlusion. To define the potential contributions of Kyn-CKA formation under clinically relevant pathological conditions, the concentrations of Kyn-CKA metabolites were determined in a humanized mouse model of SCD. Compared to non-sickle HbAA controls, homozygous HbSS mice exhibited higher hepatic TDO2 expression and elevated plasma kynurenine levels (Fig 5AC). Interestingly, a strong positive correlation was observed between kynurenine and hemin 
concentrations in HbSS mouse plasma, indicating an association between pathogenic hemin accumulation and kynurenine synthesis (Fig 5D). In this regard, while hemin failed to induce TDO2 in AML12 hepatocytes (not shown), it upregulated IDO1 expression in J774a.1 (Fig 5E). Consistent with increased Kyn-CKA formation secondary to kynurenine synthesis, urinary Cys-Kyn-CKA levels were significantly greater in $\mathrm{HbSS}$ versus HbAA mice (Fig 5F). This was recapitulated clinically in a cohort of SCD patients that, in addition to greater plasma kynurenine concentrations, also exhibited increased levels of both urinary Cys-Kyn-CKA and plasma RedKyn-CKA (Fig 5G-I). No differences in circulating tryptophan levels were observed between SCD and non-SCD subjects in either mouse or human cohorts, and kynurenine to tryptophan ratios were consistent with kynurenine pathway upregulation in SCD (Suppl Fig 4).

To test whether the anti-inflammatory actions of Kyn-CKA can modulate SCD-specific pathology, inflammatory vaso-occlusion was monitored in real-time by intravital microscopy of the pulmonary microvasculature $(16,17)$. Intravenous injection of a nanogram dose of LPS $(0.1$ $\mu \mathrm{g} / \mathrm{kg}$ ) in HbSS mice promoted formation of multicellular aggregates comprised of platelets and neutrophils that obstructed pulmonary arterioles and led to the interruption of blood flow in the lung. Pretreatment with Kyn-CKA $(10 \mathrm{mg} / \mathrm{kg}$, iv) significantly inhibited both pulmonary vasoocclusion and the size of individual multicellular aggregates (Fig 5J-L and Suppl Media). These results reinforce that Kyn-CKA formation secondary to kynurenine synthesis upregulation in SCD is an adaptive anti-inflammatory response that inhibits inflammatory events leading to vaso-occlusion and protects organs against the cumulative effects of recurrent metabolic and physical stresses. 
A

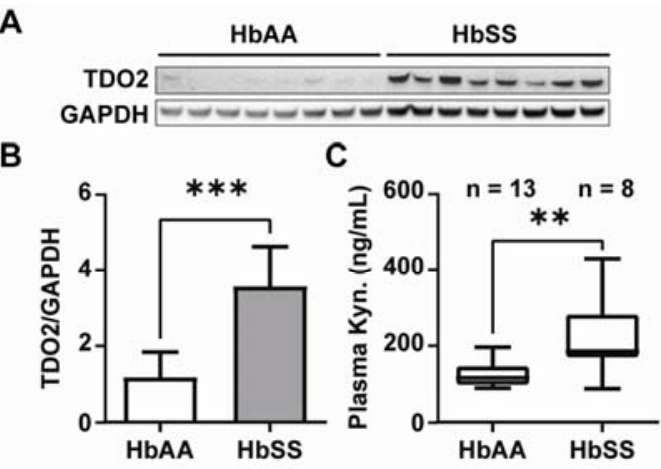

G

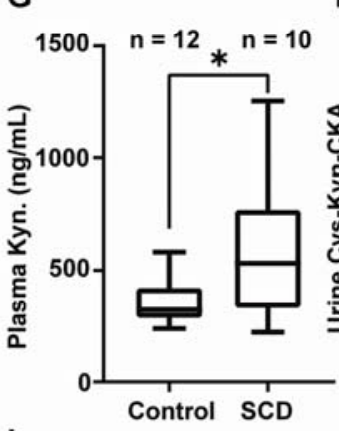

L
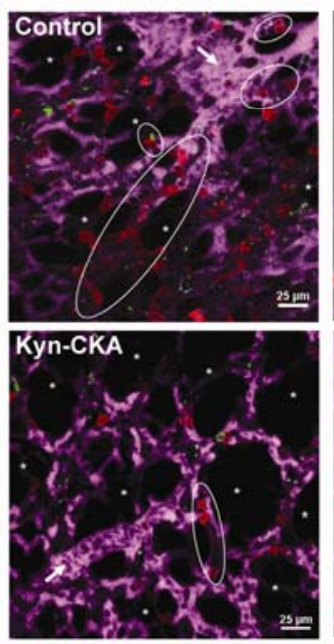

H

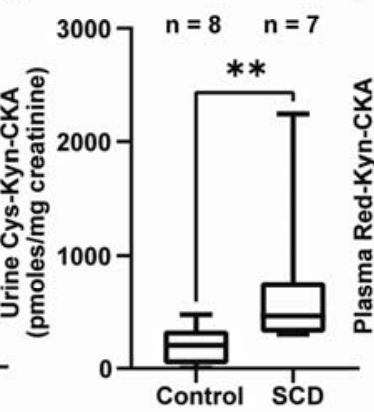

I
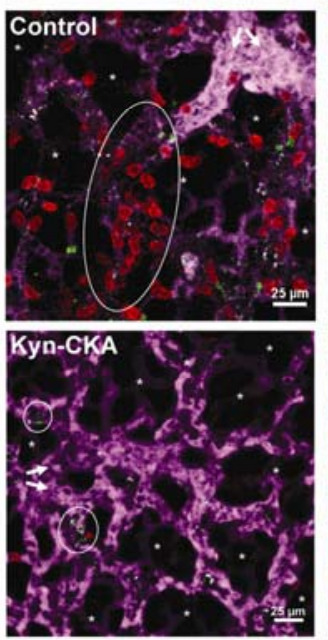

D
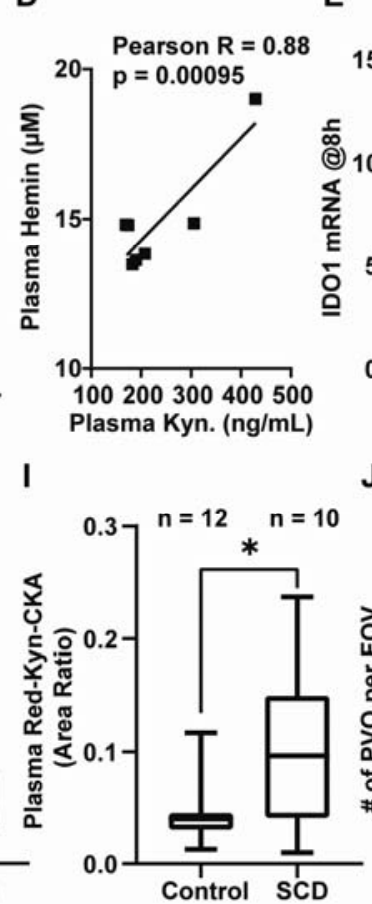

E

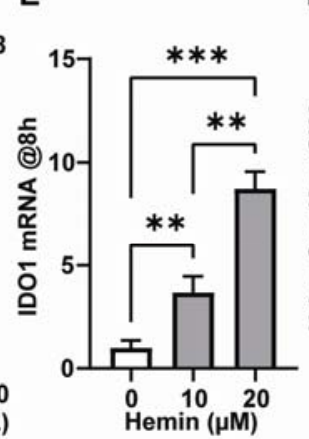

J
F

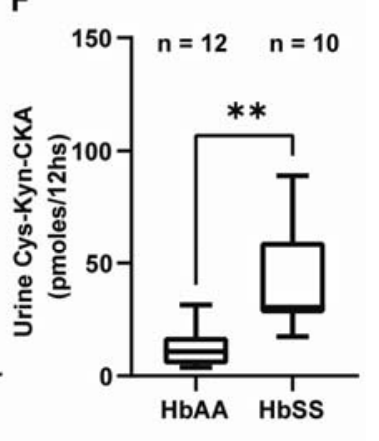

K
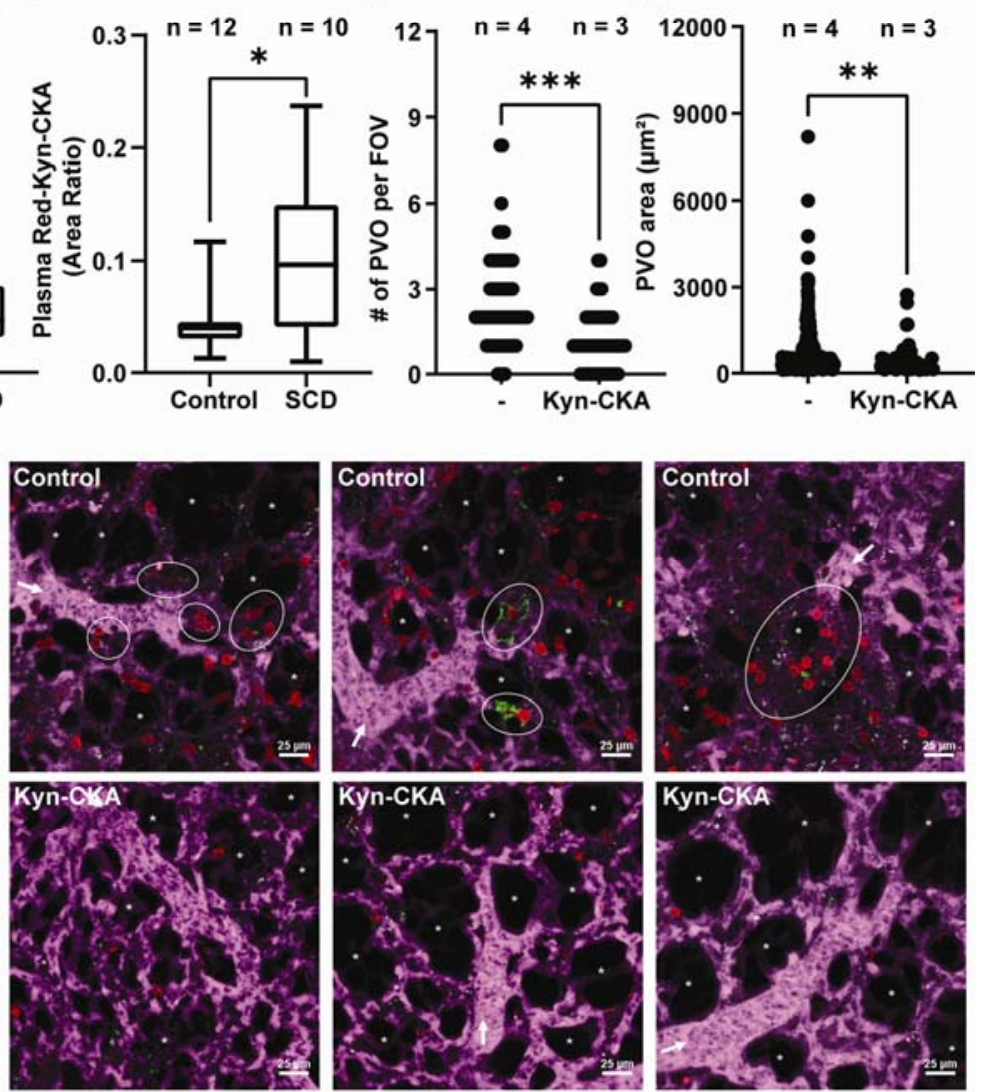

Fig. 5. Kyn-CKA synthesis is elevated in SCD and attenuates pulmonary microvascular vaso-occlusion. (A-B) TDO2 immunoblot and quantification ( $\mathrm{n}=8$ per genotype). (C) Plasma kynurenine in $\mathrm{HbAA}$ vs HbSS mice. (D) Correlation between HbSS plasma kynurenine and hemin $(n=7)$. (E) IDO1 induction by hemin in J774a.1 ( $n=3$ per condition). (F). Cys-Kyn-CKA in $12 \mathrm{~h} \mathrm{HbAA}$ and HbSS urine. (G-I) Plasma kynurenine, urine Cys-Kyn-CKA and plasma RedKyn-CKA in healthy and SCD volunteers. (J-K) Number of pulmonary vaso-occlusions per field of view and pulmonary vaso-occlusion area in HbSS mice receiving PBS or Kyn-CKA (10 $\mathrm{mg} / \mathrm{kg}$ iv) $30 \mathrm{~min}$ prior to LPS $(0.1 \mu \mathrm{g} / \mathrm{kg}$ iv). Control $\mathrm{n}=4$ (89 FOVs), Kyn-CKA $\mathrm{n}=3$ (60 FOVs). (L) Representative fields of view for HbSS mice treated as in K. Asterisks denote alveolar spaces, arrows indicate blood flow direction and dotted ellipses show pulmonary vasoocclusions. * $\mathrm{p}<0.05, * * \mathrm{p}<0.01, * * * \mathrm{p}<0.0001$ by t-test $(\mathrm{A}-\mathrm{C}, \mathrm{G}, \mathrm{I})$, Mann Whitney test $(\mathrm{F}$, $\mathrm{H}, \mathrm{J}, \mathrm{K}$ ) or one-way ANOVA and Tukey's test (E). 


\section{Discussion}

The upregulation of the rate-limiting enzymes in kynurenine metabolism is linked with diverse anti-inflammatory and immunomodulatory effects, but the precise mechanisms behind these immunosuppressive actions are incompletely understood (10). Proposed effects include TDO2- or IDO1-mediated decreases in tryptophan, alterations in $\mathrm{NAD}^{+}$availability, activityindependent signaling actions of IDO1, and the generation of bioactive kynurenine metabolites $(2,10,18)$. In particular, the activation of the aryl hydrocarbon receptor (AhR) and the G-protein coupled receptor 35 (GPR35) by kynurenine and kynurenic acid have been proposed, but whether their physiological concentrations are sufficient to engage these pathways in vivo is controversial $(19,20)$. Herein, we demonstrate that kynurenine yields a bioactive electrophile, Kyn-CKA, in vitro and in vivo. Moreover, the anti-inflammatory signaling actions of Kyn-CKA suggest that this novel metabolite could account for a significant fraction of the immunomodulatory effects ascribed to the kynurenine pathway.

A facile reaction with abundant nucleophilic thiols and amines, combined with substantial metabolic transformation, challenges the in vivo detection of endogenous electrophiles in their "free" non-adducted form, as is the case for Kyn-CKA. Strategies for electrophile discovery involve the use of highly specific LC-MS/MS and LC-HRMS detection of proximal metabolites such as non-electrophilic reduction products and excreted urinary conjugates. This approach allowed the detection of several specific Kyn-CKA metabolites in vivo: the non-electrophilic Red-Kyn-CKA in plasma, an intracellular GSH-Kyn-CKA conjugate, and the corresponding cysteine- and $\mathrm{N}$-acetyl-cysteine adducts in urine. Unequivocal identification of these metabolites is supported by co-elution with isotopically labeled standards for thiol-Kyn-CKA adducts, confirmation of molecular composition, and fragmentation analyses at the $<2 \mathrm{ppm}$ level using LC-HRMS. Importantly, the observation that Red-Kyn-CKA, Cys-Kyn-CKA, and GSH-KynCKA are present in plasma, urine, and liver extracts from untreated mice and healthy humans demonstrate that Kyn-CKA is an endogenous mediator produced in vivo under normal physiological conditions.

Electrophilic molecules such as Kyn-CKA covalently modify hyperreactive and functionally significant cysteine thiols in transcription factors, signaling proteins and enzymes to induce changes in protein and cellular function. Nucleophilic targets susceptible to electrophilic addition reactions include critical cysteines of TLR/NF- $\mathrm{KB}$ signaling, NLRP3 inflammasome components, and the Nrf2 inhibitor protein Keap1 $(11,12)$. Herein, we show both in vitro and in vivo that Kyn-CKA activates the expression of Nrf2-dependent proteins and attenuates the production of pro-inflammatory cytokines by inhibiting TLR4 and NLRP3 engagement. In the kidney, Kyn-CKA induced Nrf2-regulated gene expression, attenuated pro-inflammatory genes, and decreased the expression of the renal injury marker Kim-1 upon LPS challenge. These observations are consistent with the role of the kidney in a) kynurenine reabsorption and metabolism, b) Kyn-CKA excretion as urinary Cys-Kyn-CKA conjugates, and c) the protective effect of Nrf2 inducers against diverse renal injuries $(21,22)$. Having demonstrated the endogenous nature and robust signaling actions of Kyn-CKA, it is also shown that Kyn-CKA synthesis is upregulated under clinically relevant inflammatory conditions and impacts complex pathological responses. SCD was selected because of its chronic inflammatory nature, reports of elevated plasma kynurenine levels in patients, and a precedent for reactive electrophiles being able to modulate disease outcome $(15,23,24)$. In line with published results $(23)$, plasma kynurenine levels were elevated in both a murine SCD model and SCD patients, which was in turn associated with increased urinary Cys-Kyn-CKA and plasma Red-Kyn-CKA. Moreover, a 
strong positive correlation was observed between hemin and kynurenine levels in plasma, indicating that pathologic events in SCD promote kynurenine pathway upregulation possibly through a combination of direct and indirect effects of hemin on IDO1 and TDO2 expression (2, 25). The heightened inflammatory state of SCD results from upregulated TLR4 expression, release of pro-inflammatory hemoglobin-derived products during intravascular hemolysis, and increased gut endotoxin leakage (26-29). In this context, TLR4 activation by hemin and LPS in endothelial and immune cells and NLRP3 inflammasome engagement in platelets are critical to the onset of vaso-occlusive crises $(17,26,30,31)$. Considering the observed anti-inflammatory effects of Kyn-CKA, it was hypothesized that the upregulation of the kynurenine pathway in SCD constitutes a feedback mechanism to minimize the occurrence of vaso-occlusive events through Kyn-CKA formation. To test this concept, SCD mice were supplemented with Kyn-CKA and the formation of LPS-induced microvascular obstructions in the pulmonary circulation was quantified in real-time by intravital microscopy. Consistent with this hypothesis, increasing systemic Kyn-CKA levels before LPS challenge significantly reduced the size of neutrophil-platelet aggregates and attenuated microvascular occlusions. This effect was likely due to combined inhibitory effects on endothelial adhesion molecule expression, as well as NF- $\kappa \mathrm{B}$ and NLRP3-dependent pathway engagement in myeloid cells and platelets.

Importantly, and despite the anti-inflammatory effects of Kyn-CKA, significant associations have been found between increased plasma kynurenine levels and poor outcomes in different human diseases (6-9). However, while these findings have often led investigators to assume that kynurenine synthesis upregulation is a pathological process, evidence suggests that these responses are a part of a compensatory anti-inflammatory reaction to the specific pathological condition $(10,32)$. For instance, while genetic IDO1 ablation potentiates antitumor activity and inhibits metastasis (33), this same intervention worsens autoimmune disease (34). Furthermore, IDO1 gene transfer enhances tissue engraftment and survival in an allogenic transplantation model (35), and exogenous kynurenine administration is protective against mortality due to severe endotoxemia (2). Considering the present results, we propose that the upregulation of the kynurenine pathway is an adaptive signaling response that suppresses inflammation and protects tissues from injury via Kyn-CKA formation.

In summary, this report provides unequivocal evidence that Kyn-CKA is an endogenous mediator that contributes to the immunoregulatory effects of kynurenine and its metabolites, thus impacting disease progression in chronic inflammatory conditions. 


\section{References and Notes}

1. I. Cervenka, L. Z. Agudelo, J. L. Ruas, Kynurenines: Tryptophan's metabolites in exercise, inflammation, and mental health. Science 357, (2017). doi 10.1126/science.aaf9794

2. A. Bessede et al., Aryl hydrocarbon receptor control of a disease tolerance defence pathway. Nature 511, 184-190 (2014). doi 10.1038/nature13323

3. F. Fallarino et al., LPS-conditioned dendritic cells confer endotoxin tolerance contingent on tryptophan catabolism. Immunobiology 220, 315-321 (2015). doi 10.1016/j.imbio.2014.09.017

4. N. T. Nguyen et al., Aryl hydrocarbon receptor negatively regulates dendritic cell immunogenicity via a kynurenine-dependent mechanism. Proc Natl Acad Sci U S A 107, 19961-19966 (2010). doi 10.1073/pnas.1014465107

5. M. Hill et al., IDO expands human CD4+CD25high regulatory $\mathrm{T}$ cells by promoting maturation of LPS-treated dendritic cells. Eur J Immunol 37, 3054-3062 (2007). doi 10.1002/eji.200636704

6. H. Li et al., Metabolomic adaptations and correlates of survival to immune checkpoint blockade. Nat Commun 10, 4346 (2019). doi 10.1038/s41467-019-12361-9

7. H. Zuo et al., Plasma Biomarkers of Inflammation, the Kynurenine Pathway, and Risks of All-Cause, Cancer, and Cardiovascular Disease Mortality: The Hordaland Health Study. Am J Epidemiol 183, 249-258 (2016). doi 10.1093/aje/kwv242

8. J. Kaden, D. Abendroth, A. Volp, M. Marzinzig, Dynamics and Diagnostic Relevance of Kynurenine Serum Level after Kidney Transplantation. Ann Transplant 20, 327-337 (2015). doi 10.12659/AOT.893721

9. J. C. Schefold et al., Increased indoleamine 2,3-dioxygenase (IDO) activity and elevated serum levels of tryptophan catabolites in patients with chronic kidney disease: a possible link between chronic inflammation and uraemic symptoms. Nephrol Dial Transplant 24, 1901-1908 (2009). doi 10.1093/ndt/gfn739

10. M. Platten, E. A. A. Nollen, U. F. Rohrig, F. Fallarino, C. A. Opitz, Tryptophan metabolism as a common therapeutic target in cancer, neurodegeneration and beyond. Nat Rev Drug Discov 18, 379-401 (2019). doi 10.1038/s41573-019-0016-5

11. S. Parvez, M. J. C. Long, J. R. Poganik, Y. Aye, Redox Signaling by Reactive Electrophiles and Oxidants. Chem Rev 118, 8798-8888 (2018). doi 10.1021/acs.chemrev.7b00698

12. K. V. Swanson, M. Deng, J. P. Ting, The NLRP3 inflammasome: molecular activation and regulation to therapeutics. Nat Rev Immunol 19, 477-489 (2019). doi 10.1038/s41577-019-0165-0

13. B. D. Hood, B. Garner, R. J. Truscott, Human lens coloration and aging. Evidence for crystallin modification by the major ultraviolet filter, 3-hydroxy-kynurenine O-beta-Dglucoside. J Biol Chem 274, 32547-32550 (1999). doi 10.1074/jbc.274.46.32547

14. D. Zhang, C. Xu, D. Manwani, P. S. Frenette, Neutrophils, platelets, and inflammatory pathways at the nexus of sickle cell disease pathophysiology. Blood 127, 801-809 (2016). doi 10.1182/blood-2015-09-618538

15. G. J. Kato et al., Sickle cell disease. Nat Rev Dis Primers 4, 18010 (2018). doi 10.1038/nrdp.2018.10

16. M. F. Bennewitz et al., Lung vaso-occlusion in sickle cell disease mediated by arteriolar neutrophil-platelet microemboli. JCI Insight 2, e89761 (2017). doi 10.1172/jci.insight.89761 
17. R. Vats et al., Platelet Extracellular Vesicles Drive Inflammasome-IL-1beta-Dependent Lung Injury in Sickle Cell Disease. Am J Respir Crit Care Med 201, $33-46$ (2020). doi 10.1164/rccm.201807-1370OC

18. M. T. Pallotta et al., Indoleamine 2,3-dioxygenase is a signaling protein in long-term tolerance by dendritic cells. Nat Immunol 12, 870-878 (2011). doi 10.1038/ni.2077

19. G. Milligan, Orthologue selectivity and ligand bias: translating the pharmacology of GPR35. Trends Pharmacol Sci 32, 317-325 (2011). doi 10.1016/j.tips.2011.02.002

20. S. H. Seok et al., Trace derivatives of kynurenine potently activate the aryl hydrocarbon receptor (AHR). J Biol Chem 293, 1994-2005 (2018). doi 10.1074/jbc.RA117.000631

21. S. E. Moller, Pharmacokinetics of tryptophan, renal handling of kynurenine and the effect of nicotinamide on its appearance in plasma and urine following L-tryptophan loading of healthy subjects. Eur J Clin Pharmacol 21, 137-142 (1981). doi 10.1007/BF00637514

22. C. J. Schmidlin, M. B. Dodson, D. D. Zhang, Filtering through the role of NRF2 in kidney disease. Arch Pharm Res 43, 361-369 (2020). doi 10.1007/s12272-019-01177-2

23. S. Sabuncuoglu et al., The increased neopterin content in turkish pediatric patients with sickle cell anemia. Ann Hematol 99, 41-47 (2020). doi 10.1007/s00277-019-03817-5

24. N. Keleku-Lukwete et al., Amelioration of inflammation and tissue damage in sickle cell model mice by Nrf2 activation. Proc Natl Acad Sci U S A 112, 12169-12174 (2015). doi 10.1073/pnas.1509158112

25. G. J. Kato, M. H. Steinberg, M. T. Gladwin, Intravascular hemolysis and the pathophysiology of sickle cell disease. J Clin Invest 127, 750-760 (2017). doi 10.1172/JCI89741

26. J. D. Belcher et al., Heme triggers TLR4 signaling leading to endothelial cell activation and vaso-occlusion in murine sickle cell disease. Blood 123, 377-390 (2014). doi 10.1182/blood-2013-04-495887

27. G. Chen et al., Heme-induced neutrophil extracellular traps contribute to the pathogenesis of sickle cell disease. Blood 123, 3818-3827 (2014). doi 10.1182/blood-2013-10-529982

28. E. J. van Beers et al., Iron, inflammation, and early death in adults with sickle cell disease. Circ Res 116, 298-306 (2015). doi 10.1161/CIRCRESAHA.116.304577

29. D. Zhang et al., Neutrophil ageing is regulated by the microbiome. Nature 525, 528-532 (2015). doi 10.1038/nature15367

30. S. Vogel et al., The platelet NLRP3 inflammasome is upregulated in sickle cell disease via HMGB1/TLR4 and Bruton tyrosine kinase. Blood Adv 2, 2672-2680 (2018). doi 10.1182/bloodadvances.2018021709

31. J. D. Beckman et al., Endothelial TLR4 Expression Mediates Vaso-Occlusive Crisis in Sickle Cell Disease. Front Immunol 11, 613278 (2020). doi 10.3389/fimmu.2020.613278

32. W. D. Hedrich, H. Wang, Friend or Foe: Xenobiotic Activation of Nrf2 in Disease Control and Cardioprotection. Pharm Res 38, 213-241 (2021). doi 10.1007/s11095-02102997-y

33. X. Zheng et al., Reinstalling antitumor immunity by inhibiting tumor-derived immunosuppressive molecule IDO through RNA interference. J Immunol 177, 56395646 (2006). doi 10.4049/jimmunol.177.8.5639

34. S. Y. Chen et al., Amelioration of rat collagen-induced arthritis through CD4+ T cells apoptosis and synovial interleukin-17 reduction by indoleamine 2,3-dioxygenase gene therapy. Hum Gene Ther 22, 145-154 (2011). doi 10.1089/hum.2009.217

35. K. Iken et al., Indoleamine 2,3-dioxygenase and metabolites protect murine lung allografts and impair the calcium mobilization of T cells. Am J Respir Cell Mol Biol 47, 405-416 (2012). doi 10.1165/rcmb.2011-0438OC 


\section{Funding:}

National Institutes of Health grant K01HL133331 (DAV)

National Institutes of Health grant R03HL157878 (DAV)

National Institutes of Health grant P30DK079307 (DAV)

National Institutes of Health grant GM125944 (FJS)

National Institutes of Health grant DK112854 (FJS)

National Institutes of Health grant R01HL 133864 (ACS)

National Institutes of Health grant R01HL 128304 (ACS)

National Institutes of Health grant R01HL 149824 (ACS)

American Heart Association grant 19EIA34770095 (ACS)

National Institutes of Health grant R01HL133864 (GJK)

National Institutes of Health grant R01MD009162 (GJK)

National Institutes of Health grant U54HL141011 (SO)

National Institutes of Health grant R01HL128297 (PS)

National Institutes of Health grant R01HL141080 (PS)

This publication was also made possible by support from the Vascular Medicine Institute, the Hemophilia Center of Western Pennsylvania, the Institute for Transfusion Medicine and Pittsburgh Liver Research Center (NIH grant P30DK120531).

\section{Author contributions:}

Conceptualization: DAV, MC, MFP, GJK, PS, FJS

Methodology: DAV, MC, MFP, SRS, SGW, TB, PS

Investigation: DAV, MC, MFP, SRW, TB, SG, MD, NC, SY

Funding acquisition: DAV, SOF, BAF, SOF, FJS, ACS, PS

Project administration: DAV

Resources: DAV, GJK, SG, SGW, SOF, BAF, FJS, PS

Supervision: DAV, ACS, PS, GJK, SOF, FJS, BAF

Writing - original draft: DAV, MC, MFP

Writing - review \& editing: DAV, BAF, PS, FJS

Competing interests: Authors declare that they have no competing interests. GJK is an employee of CSL Behring.

Data and materials availability: All data are available in the main text or the supplementary materials. 
bioRxiv preprint doi: https://doi.org/10.1101/2022.02.08.479451; this version posted February 10, 2022. The copyright holder for this preprint

(which was not certified by peer review) is the author/funder, who has granted bioRxiv a license to display the preprint in perpetuity. It is made available under aCC-BY-NC-ND 4.0 International license.

\section{Supplementary Materials}

Materials and Methods

Figs. S1 to S4

Movies Control-1 to 5, and Kyn-CKA-1 to 5 